Supporting Information for

\title{
Graphene-Foam Cantilever Produced via Simultaneous Foaming and Doping Effect of Organic Coagulant
}

Sung Hyun Noh, ${ }^{a}$ Hun Park, ${ }^{b}$ Wonsik Eom, ${ }^{a}$ Hak Bong Lee, ${ }^{a}$ Dong Jun Kang, ${ }^{a}$ Jae Yong Cho, ${ }^{c}$ Tae Hyun Sung, ${ }^{c}$ and Tae Hee Han ${ }^{* a}$

aDepartment of Organic and Nano Engineering, Hanyang University, Seoul, 04763, Republic of Korea

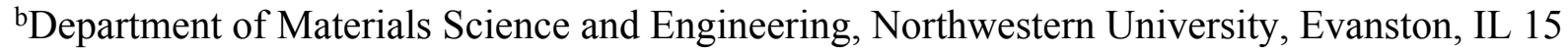
60208, USA

${ }^{c}$ Department of Electrical Bio-Engineering, Hanyang University, Seoul, 04763, Republic of Korea

\section{Corresponding Author}

E-mail: than@hanyang.ac.kr (T.H.H.) 
(a)

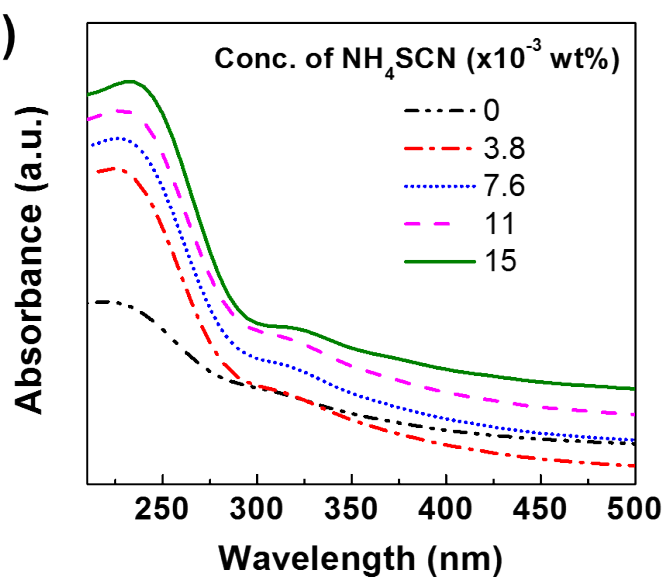

(b)

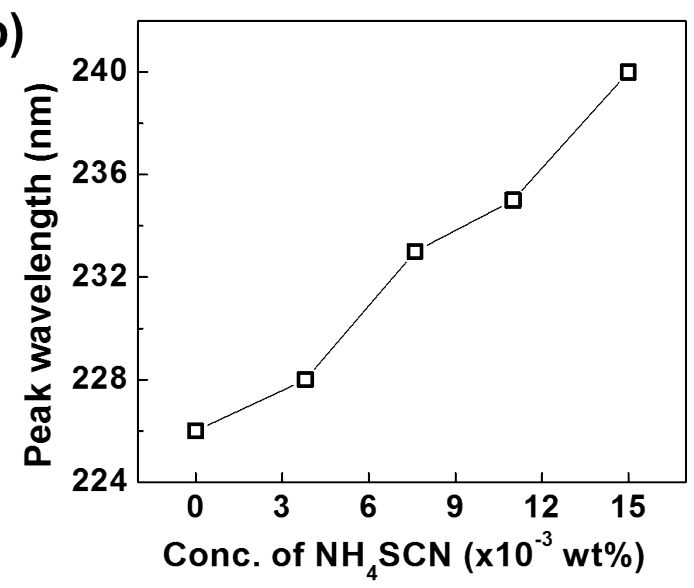

Figure S1. (a) UV-vis absorption spectra of diluted GO solution under various $\mathrm{NH}_{4} \mathrm{SCN}$ concentrations. (b) The shift of $\pi \rightarrow \pi^{*}$ peak location for each $\mathrm{NH}_{4} \mathrm{SCN}$ concentrations obtained from UV-vis spectra in (a).

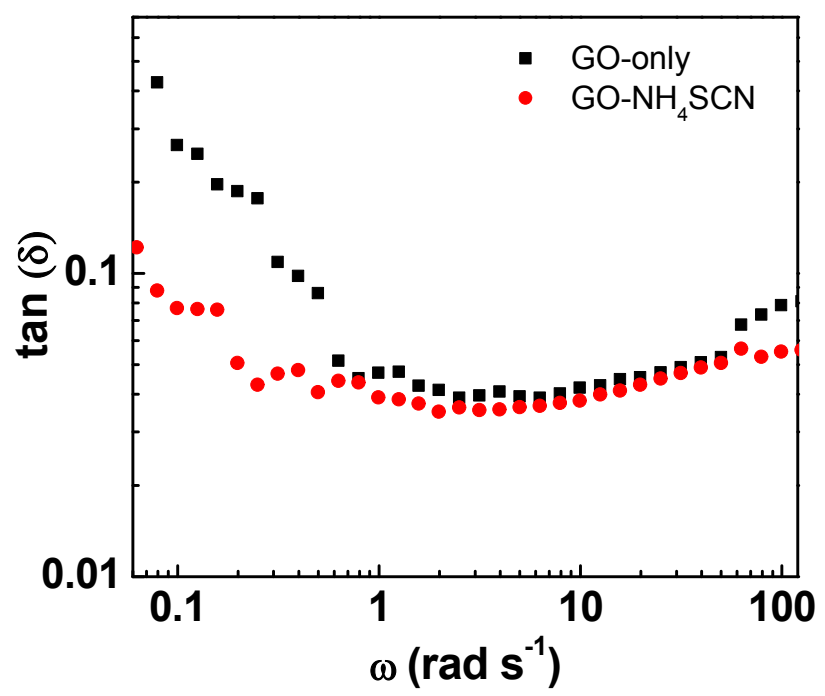

Figure S2. Plot of $\tan \delta$ comparison for GO-only and GO-NH${ }_{4} \mathrm{SCN}$. 
(a)

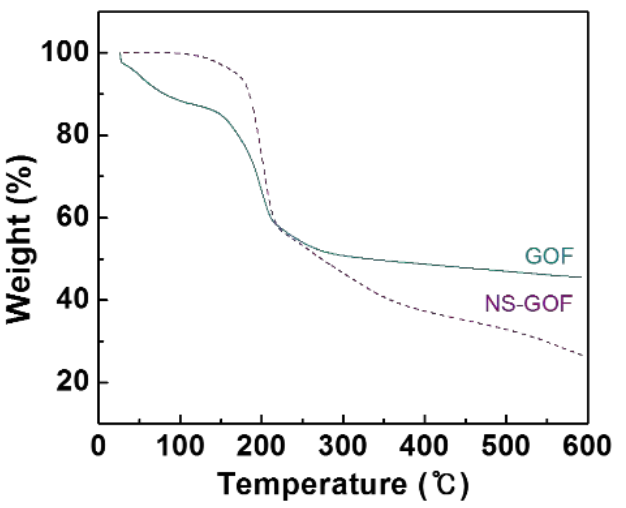

(b)

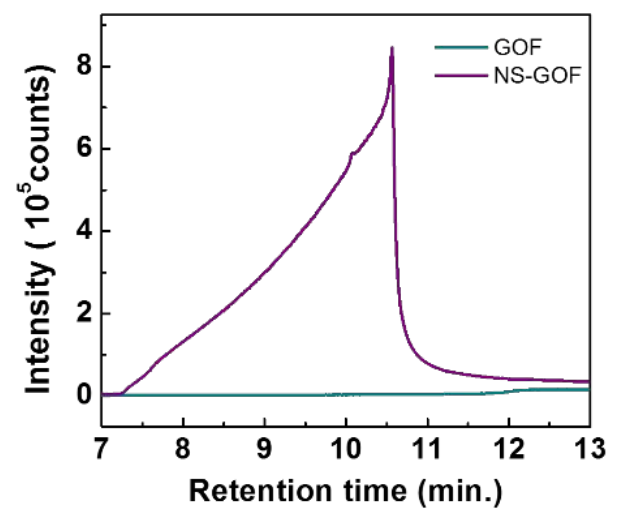

Figure S3. (a) TGA measurement of GOF and NS-GOF performed under inert atmosphere. (b) GC-MS results of GOF and NS-GOF.

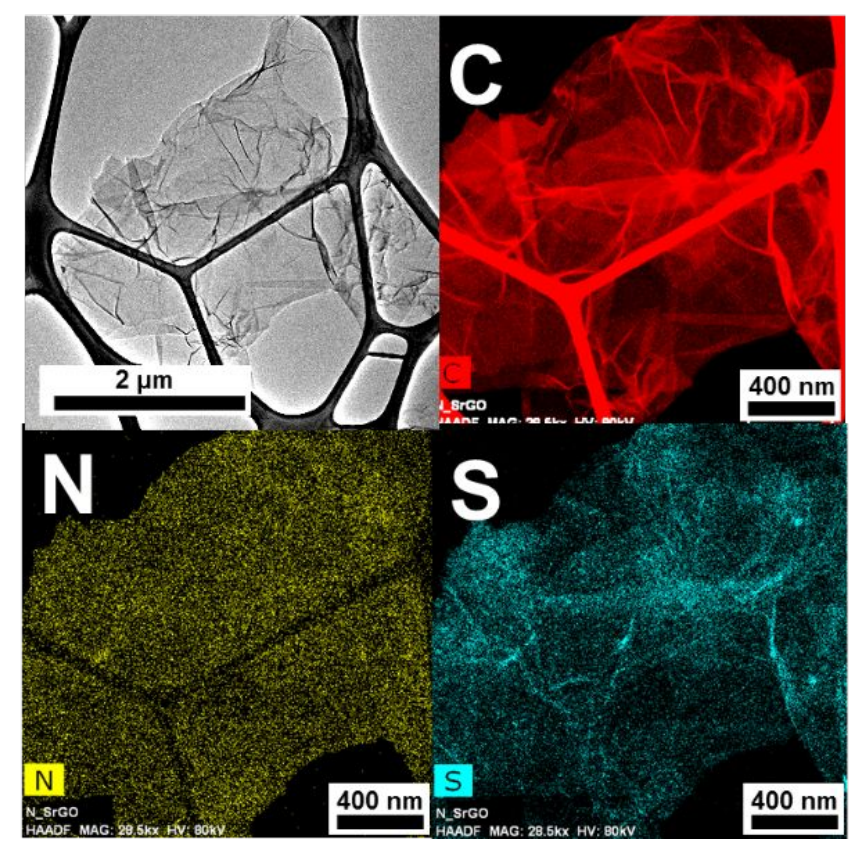

Figure S4. EDX mapping analysis of NS-GF sheets observed by TEM. 


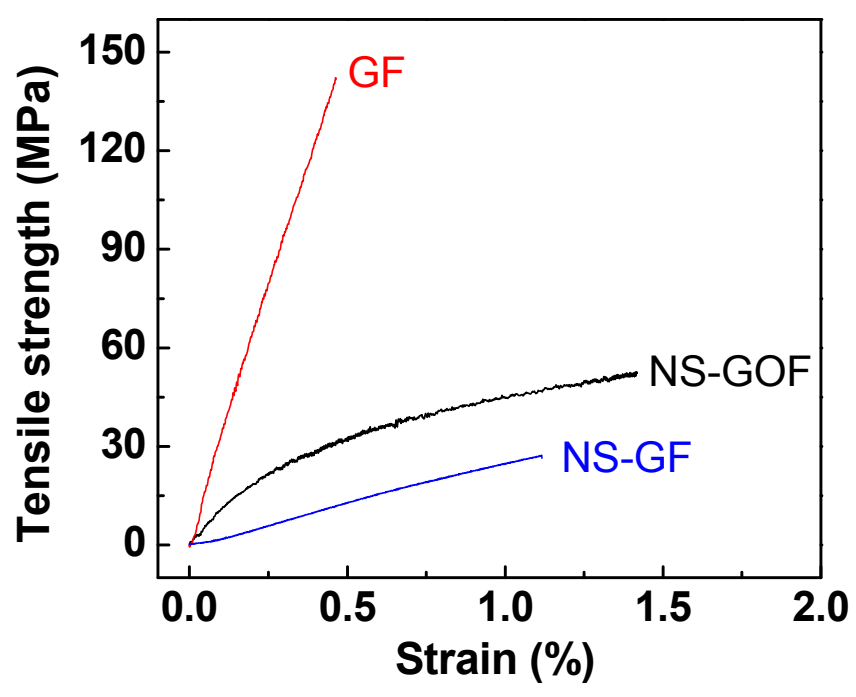

Figure S5. Stress-strain curve results of GF, NS-GOF and NS-GF.
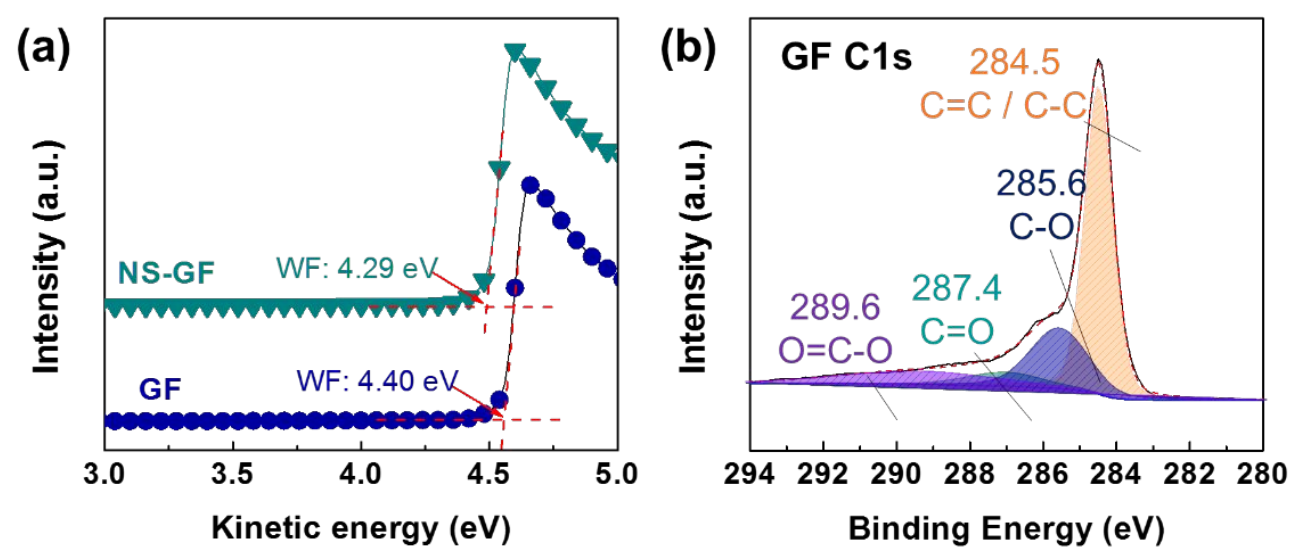

Figure S6. (a) UPS spectra of NS-GF and GF. (b) Peak deconvoluted C1s spectrum of GF. 


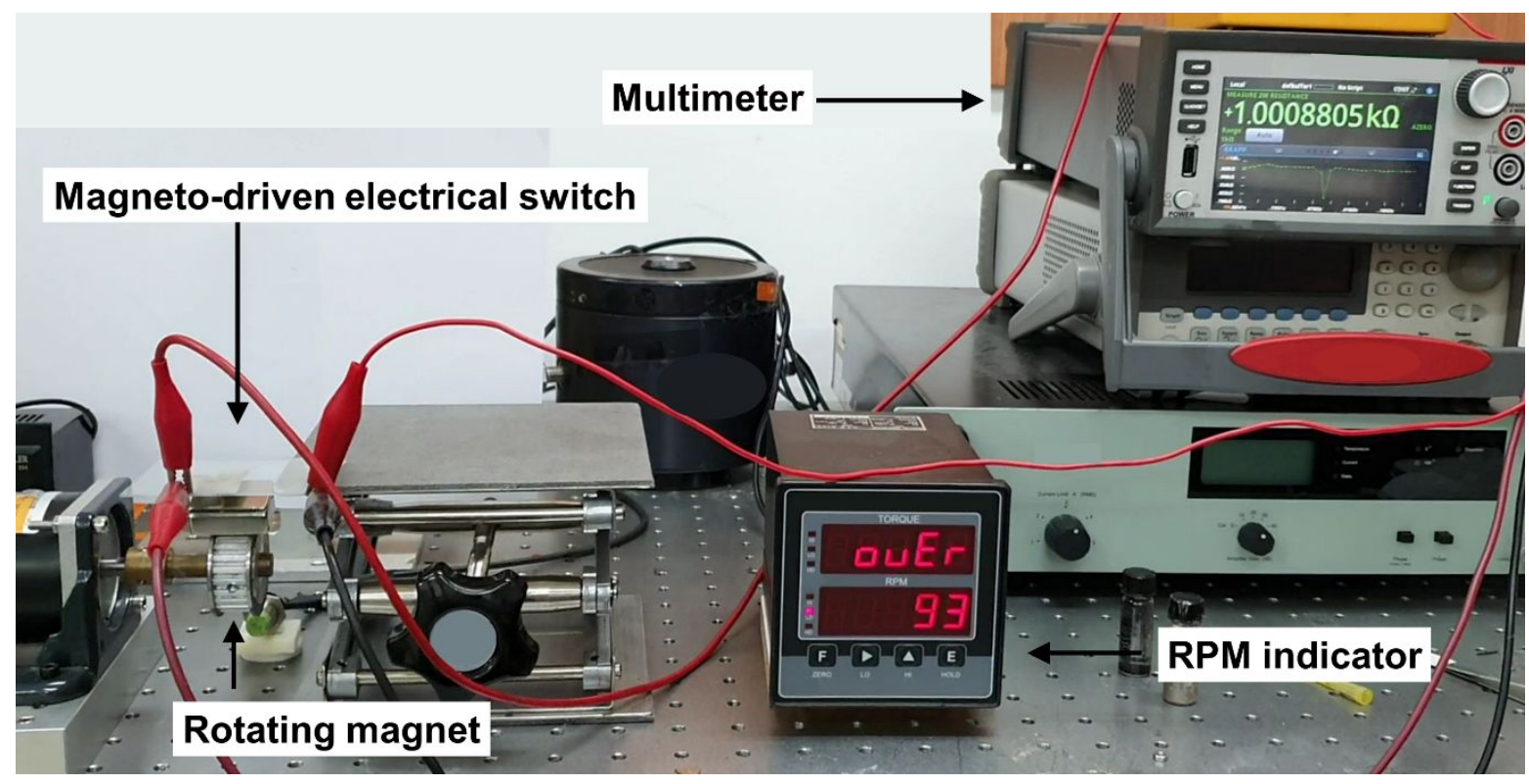

Figure S7. Experimental setup image of our NS-GF cantilever magneto-actuator switch.

Table S1. Table summarizing the identified gases during the GC-MS analysis of GOF and NSGOF.

\begin{tabular}{c|c}
\hline GOF gases & NS-GOF gases \\
\hline Dimethyl ether & Carbonyl sulfide \\
Allen & Carbon disulfide \\
Carbamic acid & Hydrogen azide \\
1,2-Propadiene-1,3-dione & Sulfur dioxide \\
2-Butanone & Hydrogen azide \\
Methoxy acetic acid & N-methyl formamide \\
\hline
\end{tabular}

